\title{
Méthode de réduction de modèles pour l'analyse de structures composites à matrice élastomérique
}

\author{
Stéphane Lejeunes - Adnane Boukamel - Bruno Cochelin \\ Ecole Centrale de Marseille \\ IMT/Technopôle Chateau-Gombert, F-13451 Marseille cedex 20 \\ Laboratoire de Mécanique et d'Acoustique de Marseille \\ 31 chemin Joseph-Aiguier, F-13402 Marseille cedex 20 \\ \{stephane.lejeunes, adnane.boukamel,bruno.cochelin\}@ec-marseille.fr
}

\begin{abstract}
RÉSUMÉ. On propose, dans ce papier, une technique pour résoudre les problèmes d'équilibre de structures tridimensionnelles possédant des propriétés d'invariance selon une direction et dont le comportement présente des non-linéarités géométriques et matérielles. Basée sur une formulation en éléments finis, elle consiste à projeter sur une base polynomiale, bien choisie visà-vis des propriétés d'invariance, les champs inconnus permettant ainsi de réduire la dimension du problème à résoudre. Cette technique de réduction de modèle a été mise en cuvre pour l'analyse d'une structure poutre à base élastomérique avec une loi de comportement viscohyperélastique quasi incompressible.

ABSTRACT. In this paper we present a model reduction technique, to find the equilibrium state at finite strain of tridimensional structures which have, invariant properties in a direction. Based on finite-elements formulation, this technique consists in the projection of the unknows fields on a polynomial basis in the purpose to reduce the problem dimension. A finite-element is developped for the case of nearly-incompressible visco-hyperelastic behavior for the study of an elastomeric based beam structure.

MOTS-CLÉS : réduction de modèle, visco-hyperélasticité, éléments finis, élastomère, incompressibilité.

KEYWORDS: model reduction, visco-hyperelasticity, finite elements, rubber, incompressibility.
\end{abstract}

DOI:10.3166/REMN.16.795-811 (c) 2007 Lavoisier, Paris

REMN - 16/2007. Modélisations numériques en mécanique, pages 795 à 811 


\section{Introduction}

La motivation industrielle de ce papier s'articule principalement autour de l'étude d'une structure composite à matrice élastomérique, ayant vocation à être utilisée dans l'aéronautique. L' « Elastomeric Flex Beam » (E.F.B.) est l'exemple type de structure nécessitant une stratégie de modélisation adaptée pour réduire la taille ou le temps de calcul du modèle (le modèle 3D conduisant à plus de 5 millions de ddl). Il s'agit d'une poutre en élastomère renforcée par des tiges constituées de deux matériaux isotropes (voir figure 1).

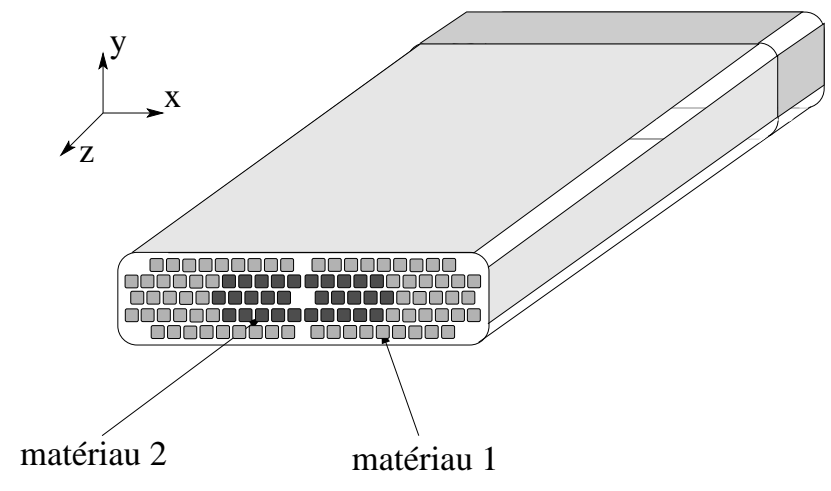

Figure 1. Poutre composite EFB

De manière générale, on peut distinguer dans la littérature trois catégories de méthodes permettant d'obtenir une réduction, de taille ou de temps de calcul, de ce type de problème. La première concerne la recherche de modèles de comportement équivalents, qui sont le plus souvent obtenus par homogénéisation. On peut ainsi rencontrer des méthodes d'homogénéisation périodique, linéaires (Dumontet, 1990) ou non linéaires (Devries, 1998; Lahellec et al., 2003). Ce type de méthode a déjà été employé pour la poutre E.F.B. en considérant un comportement linéaire de l'élastomère (Delorme, 1997). Des méthodes de parallélisations ont également été proposées en utilisant une approche par sous-domaines (Léné et al., 2001), ou multiniveau dans le cas de la poutre E.F.B. (Meo et al., 2002). Enfin, dans le cas non linéaire, il existe un certain nombre d'éléments finis spécifiques qui peuvent permettre de diminuer la taille d'un modèle. Les éléments finis hiérarchiques et notament la p-méthode rentrent dans cette dernière catégorie (Duster et al., 2003), ainsi que les éléments pseudoaxisymétriques qui permettent à l'aide de séries de Fourier de traiter le cas de structures à symétrie de révolution sous chargement non symétrique (Boukamel, 1988).

Nos travaux s'inscrivent dans cette dernière catégorie de méthodes et héritent d'une logique datant du milieu des années 1970, inspirée des travaux de Cheung concernant les éléments finis semi-analytiques pour la modélisation des plaques élastiques ; « finite strip method» initialement utilisées pour des applications en génie civil (Cheung, 1968), puis étendues à d'autres structures (Cheung et al., 1995; Cheung et 
al., 2001). En effectuant une projection des inconnues du problème (champs de déplacements et de pression) sur une base polynomiale, on peut condenser, dans l'élémentfini, une ou plusieurs directions géométriques. Ce type d'approche a été mise en œuvre, dans des travaux récents, pour des structures lamifiées élastomère-métal, en considérant un comportement hyperélastique quasi incompressible de l'élastomère (Lejeunes et al., 2006). L'avantage de ce type de méthode réside dans sa capacité à obtenir une solution de bonne qualité en termes de comportement global (raideur équivalente d'une structure), mais aussi local (champs de contraintes ou de déformations) avec un faible nombre de degrés de liberté. Par ailleurs, on peut noter que ce type de stratégie continue de connaître un succès croissant, notamment dans le domaine du génie civil, voir par exemple (Choi et al., 2002; Davenne et al., 2003).

Dans cet article, nous proposons d'appliquer ce type de stratégie à un modèle de comportement visco-hyperélastique quasi incompressible de Kelvin-Voigt à viscosité non linéaire. Après une présentation de la formulation variationnelle adoptée pour la modélisation de ce type de comportement, nous détaillons la décomposition permettant une réduction 3D-2D du problème. La mise en œuvre de l'élément fini réduit est ensuite introduite, suivie d'une validation par la confrontation des résultats du modèle réduit avec un modèle 3D de référence. Enfin un cas de charge représentatif (sur l'E.F.B.) est étudié à l'aide d'un modèle réduit.

\section{Comportement visco-hyperélastique de Kelvin-Voigt}

Dans le cadre d'une description lagrangienne, on désigne par $\mathbf{F}=\partial \mathbf{x} / \partial \mathbf{X}$ le tenseur gradient décrivant le mouvement local, $\mathbf{C}=\mathbf{F}^{T} \mathbf{F}$ le tenseur de Cauchy Green gauche et $\dot{\mathbf{C}}=d \mathbf{C} / d t$. La loi de comportement d'un modèle de Kelvin-Voigt est obtenue en généralisant le modèle rhéologique de la figure 2 aux grandes déformations. Dans le cas isotherme, les potentiels d'énergie libre et de pseudo-dissipation (notés $\psi$ et $\varphi$ ) peuvent être supposés comme dépendant respectivement de $\mathbf{C}$ et de $\dot{\mathbf{C}}$.

$\psi(\mathbf{C})$

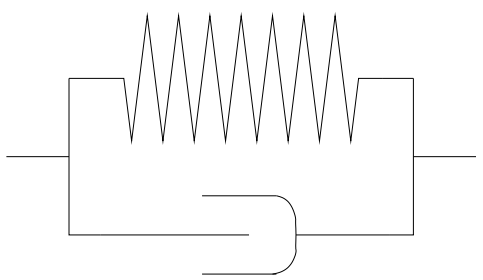

$\varphi(\dot{\mathbf{C}})$

Figure 2. Modèle de Kelvin-Voigt 
En utilisant les deux principes fondamentaux de la thermodynamique, on peut obtenir une forme générale de la loi de comportement, exprimée en fonction du premier tenseur des contraintes de Piola-Kirchhoff (noté $\Pi$ ) :

$$
\boldsymbol{\Pi}=2 \mathbf{F} \frac{\partial \psi}{\partial \mathbf{C}}+2 \mathbf{F} \frac{\partial \varphi}{\partial \dot{\mathbf{C}}}-p \mathbf{C o f} \mathbf{F}=\boldsymbol{\Pi}_{h y p}+\boldsymbol{\Pi}_{v i s c}-p \mathbf{C o f} \mathbf{F}
$$

où Cof $\mathbf{F}$ désigne la matrice des coffacteurs de $\mathbf{F}$.

La variable $p$ est un multiplicateur de Lagrange assimilable à une pression afin de tenir compte de la condition d'incompressibilité :

$$
J=\operatorname{det} \mathbf{F}=1
$$

\subsection{Contrainte hyperélastique}

L'élastomère étudié a un comportement isotrope quasi incompressible, pour le représenter nous avons choisi d'utiliser un modèle polynomial à cinq termes de James et al. (1975). Il s'agit d'un modèle phénoménologique fonction des deux premiers invariants fondamentaux de $\mathbf{C}$ (notés $I_{1}, I_{2}$ ), ce modèle étant un cas particulier de la série proposée par Rivlin et al. (1951). Le potentiel d'énergie libre s'écrit dans ce cas :

$$
\begin{aligned}
\psi\left(I_{1}, I_{2}\right)=C_{10}\left(I_{1}-3\right) & +C_{01}\left(I_{2}-3\right)+C_{11}\left(I_{1}-3\right)\left(I_{2}-3\right) \\
& +C_{20}\left(I_{1}-3\right)^{2}+C_{02}\left(I_{2}-3\right)^{2}
\end{aligned}
$$

En notant $\psi_{i}$ la différenciation de $\psi\left(I_{1}, I_{2}\right)$ par rapport à $I_{i}$ (avec $\left.i=1,2\right)$, on obtient la partie hyperélastique du tenseur des contraintes :

$$
\boldsymbol{\Pi}_{h y p}=2 \mathbf{F} \frac{\partial \psi}{\partial \mathbf{C}}=2 \mathbf{F}\left[\left(\psi_{1}+\psi_{2} I_{1}\right) \mathbf{I}-\psi_{2} \mathbf{C}\right]
$$

avec I le tenseur identité d'ordre 2.

\subsection{Contrainte visqueuse non linéaire}

En postulant une forme non linaire du pseudopotentiel de dissipation telle que $\left(\eta_{0}\right.$ et $n$ étant des coefficients matériaux à identifier) :

$$
\varphi(\dot{\mathbf{C}})=\frac{\eta_{0}}{n}\|\dot{\mathbf{C}}\|^{n} \quad n>0
$$

la norme utilisée correspond à : $\|\dot{\mathbf{C}}\|=(\dot{\mathbf{C}}: \dot{\mathbf{C}})^{1 / 2}$, on obtient donc la partie visqueuse des contraintes :

$$
\Pi_{v i s c}=2 \mathbf{F} \frac{\partial \varphi}{\partial \dot{\mathbf{C}}}=2 \eta_{0}\|\dot{\mathbf{C}}\|^{n-1} \mathbf{F} \dot{\mathbf{C}}
$$




\subsection{Formulation variationnelle en lagrangien perturbé}

La forme variationnelle, que nous avons utilisée pour écrire le problème d'équilibre mécanique, fait partie des méthodes multichamps. Elle hérite du principe variationnel de Herrmann qui est une particularisation du principe de Hellinger-Reissner pour le cas des matériaux élastiques incompressibles (Herrmann, 1964). On considère donc deux inconnues qui sont le champ de déplacement; $\mathbf{u}$ et le multiplicateur de Lagrange permettant d'imposer la condition d'incompressibilité ; $p$.

On note $\Omega_{0}$ le domaine occupé par le solide dans la configuration initiale, $\partial \Omega_{u}$ et $\partial \Omega_{f}$ les parties du contour où sont appliqués des déplacements imposés ou des efforts imposés. On définit également les espaces de solutions $\mathcal{V} \times \mathcal{Q}$ et des champs virtuels, notés $(\delta \mathbf{u}, \delta p) \in \mathcal{V}_{0} \times \mathcal{Q}$, tels que :

$$
\begin{aligned}
\mathcal{V} & =\left\{\mathbf{u} \in\left(H^{1}\left(\Omega_{0}\right)\right)^{3}, \mathbf{u}=\mathbf{u}_{\text {ext }} \quad \text { sur } \quad \partial \Omega_{u}\right\} \\
\mathcal{V}_{0} & =\left\{\mathbf{u} \in\left(H^{1}\left(\Omega_{0}\right)\right)^{3}, \mathbf{u}=0 \text { sur } \partial \Omega_{u}\right\} \\
\mathcal{Q} & =\left\{p \in L^{2}\left(\Omega_{0}\right)\right\}
\end{aligned}
$$

La formulation variationnelle obtenue en utilisant le principe des puissances virtuelles avec une perturbation de la contrainte d'incompressibilité, nous conduit au problème suivant :

$$
\begin{aligned}
& \text { Trouver }(\mathbf{u}, p) \in \mathcal{V} \times \mathcal{Q} \forall(\delta \mathbf{u}, \delta p) \in \mathcal{V}_{0} \times \mathcal{Q} \\
& \begin{cases}\int_{\Omega_{0}}\left(\boldsymbol{\Pi}_{h y p}(\mathbf{u})+\mathbf{\Pi}_{v i s c}(\mathbf{u})-p \operatorname{Cof} \mathbf{F}(\mathbf{u})\right) & : \nabla \delta \mathbf{u}-\int_{\Omega_{0}} \mathbf{f} . \delta \mathbf{u} d V \\
\int_{\Omega_{0}}(-(J(\mathbf{u})-1)-\alpha p) \delta p d V=0 & -\int_{\partial \Omega_{f}} \mathbf{T} . \delta \mathbf{u} d S=0\end{cases}
\end{aligned}
$$

avec $\mathbf{f}$ et $\mathbf{T}$ les efforts volumiques et surfaciques exercés sur le domaine $\Omega_{0}$ et le contour $\partial \Omega_{f}, \alpha$ le coefficient de perturbation pouvant être vu comme l'inverse d'un module de compressibilité.

\section{Mise en ouvre de la technique de réduction de modèles}

\subsection{Approximation des champs variables}

L'idée de départ consiste à construire des éléments finis réduits, basés sur des fonctions de formes suffisamment riches pour approximer l'évolution des champs de déplacement $\mathbf{u}$ et de pression $p$ dans la direction d'élancement. Pour une structure de type poutre cela revient à discrétiser par éléments finis uniquement une section transverse en ayant au préalable projeté les champs sur une base de fonctions continues et régulières (voir figure 3). 


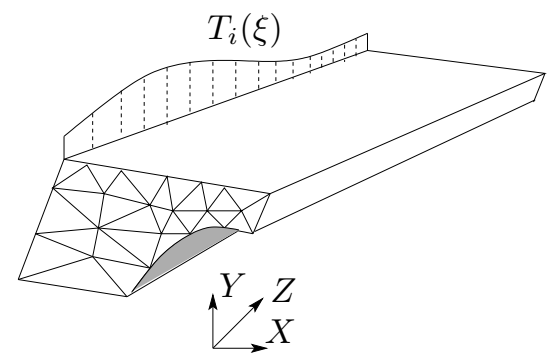

Figure 3. Réduction $3 D-2 D$

Ainsi, dans le cas d'une réduction 3D-2D, en utilisant une base polynomiale $\mathcal{T}$, on obtient avec le paramétrage $\xi=2 Z / L$ ( $L$ étant la longueur dans la direction de l'élancement) :

$$
\mathbf{u}(X, Y, Z)=\sum_{i=0}^{n_{u}} \mathbf{u}^{i}(X, Y) T_{i}(\xi), \quad p(X, Y, Z)=\sum_{i=0}^{n_{p}} p^{i}(X, Y) T_{i}(\xi)
$$

$n_{u}$ et $n_{p}$ étant les ordres d'approximations des champs dans la base polynomiale $\mathcal{T}$.

Le choix de cette base de projection doit être guidé par le type de sollicitations à traiter et doit permettre de décrire l'évolution des grandeurs mécaniques suivant la direction condensée $(Z)$, tout en traduisant les effets de bords au voisinage des sections extrêmes. Ainsi, nous utilisons les polynômes de Lagrange d'ordre 1 (permettant d'imposer directement les conditions cinématiques de type translations et/ou rotations des sections extrêmes), enrichis par des fonctions bulles, constituant une correction de l'évolution des grandeurs mécaniques dans la direction condensée. Soit de façon formelle, en utilisant les polynômes de Legendre $L_{n}(\xi)$ :

$$
T_{0}(\xi)=\frac{1-\xi}{2}, \quad T_{1}(\xi)=\frac{1+\xi}{2}, \quad T_{n}(\xi)=\frac{L_{n}(\xi)-L_{n-2}(\xi)}{\sqrt{2(2 i-1)}}
$$

Cette base présente des propriétés d'orthonormalité des dérivées premières, ainsi qu'une quasi orthogonalité des membres de la base pour le produit scalaire de $L_{2}([-1,1])$ (Cugnon, 2000). Ces propriétés particulières ont une influence sur le conditionnement de l'opérateur tangent (Lejeunes et al., 2006).

\subsection{Discrétisation par éléments finis}

A partir de la décomposition des champs inconnus présentée avec les équations [11], on peut réaliser une approximation éléments finis 2D classique du déplacement 
et de la pression en utilisant des fonctions de formes basées sur des polynômes de Lagrange, on a,

$$
\begin{aligned}
& \mathbf{u}_{h}(X, Y, Z)=\sum_{i=0}^{n_{u}} T_{i}(\xi) \sum_{j=1}^{l_{u}} N_{u}^{j}(X, Y) \mathbf{u}_{j}^{i} \\
& p_{h}(X, Y, Z)=\sum_{i=0}^{n_{p}} T_{i}(\xi) \sum_{j=1}^{l_{p}} N_{p}^{j}(X, Y) p_{j}^{i}
\end{aligned}
$$

$N_{u}^{j}$ et $N_{p}^{j}$ sont les polynômes d'interpolations respectifs du déplacement et de la pression.

Ainsi, les degrés de liberté pour les nœuds de déplacement sont les composantes $\mathbf{u}_{j}^{i}$ du déplacement dans la base $\mathcal{T}$, alors que les composantes $p_{j}^{i}$ de la pression constituent les degrés de liberté des nœuds de pression.

On note $[B]$ l'opérateur gradient élémentaire qui permet de construire $\mathbf{F}$ à partir des degrés de liberté élémentaires :

$$
\mathbf{F}=[B]\left\{u_{j}^{i}\right\}+\mathbf{I} \quad i=0, \ldots, n_{u} \quad j=0, \ldots, l_{u}
$$

En utilisant un schéma explicite d'ordre 1 en temps, on peut approximer $\dot{\mathbf{C}}$ au temps $t$ (connaissant $\mathbf{C}$ au temps $t-\Delta t$ ) par :

$$
\dot{\mathbf{C}}^{(t)}=\frac{1}{\Delta t}\left(\mathbf{C}^{(t)}-\mathbf{C}^{(t-1)}\right)
$$

La contrainte visqueuse au temps $t$ s'écrit donc de la manière suivante :

$$
\boldsymbol{\Pi}_{\text {visc }}^{(t)}=2 \eta_{0}\left\|\dot{\mathbf{C}}^{(t)}\right\|^{n} \mathbf{F}^{(t)} \dot{\mathbf{C}}^{(t)}
$$

La linéarisation des équations [10] à l'aide d'un schéma de Newton-Rapshon, nous conduit au système matriciel suivant (au temps $t$ ) :

$$
\mathcal{A}_{e=1}^{n e l}<\Delta u_{e}, \Delta p_{e}>\left(\left[\begin{array}{cc}
K_{t} & G \\
G^{t} & \alpha I
\end{array}\right]\left\{\begin{array}{c}
\Delta u_{e} \\
\Delta p_{e}
\end{array}\right\}+\left\{\begin{array}{c}
r_{u} \\
r_{p}
\end{array}\right\}\right)=0
$$

avec $\mathcal{A}$ l'opérateur d'assemblage. 
En notant $\Omega_{e}$ le domaine élémentaire, les matrices et vecteurs élémentaires sont définis par :

$$
\begin{aligned}
{\left[K_{t}\right] } & =\int_{\Omega_{e}}[B]^{T}\left[\mathbb{C}_{h y p}\left(\mathbf{u}_{h}\right)+\mathbb{C}_{v i s c}\left(\mathbf{u}_{h}, \mathbf{C}^{t-1}\right)-p_{h} \frac{\partial \mathbf{C o f} \mathbf{F}}{\partial \mathbf{F}}\right][B] d \Omega \\
{[G] } & =-\int_{\Omega_{e}} \sum_{i=0}^{n_{p}} T_{i}(\xi)\left\{N_{p}\right\}<\operatorname{Cof} \mathbf{F}>d \Omega \\
{[\alpha I] } & =-\alpha \int_{\Omega_{e}} \sum_{i=0}^{n_{p}} \sum_{j=0}^{n_{p}} T_{i}(\xi) T_{j}(\xi)\left\{N_{p}\right\}<N_{p}>d \Omega \\
\left\{r_{u}\right\} & =\int_{\Omega_{e}}[B]^{T}\left[\Pi_{h y p}\left(\mathbf{u}_{h}, \mathbf{p}_{h}\right)+\Pi_{v i s c}\left(\mathbf{u}_{h}, \mathbf{C}^{t-1}\right)\right] d \Omega \\
& -\int_{\Omega_{e}} \sum_{i=0}^{n_{u}} \sum_{j=0}^{n_{u}} T_{i}(\xi) T_{j}(\xi)\left\{N_{u}\right\}<f_{j}^{e x t}>d \Omega \\
& -\int_{\Omega_{e} \cap \partial \Omega_{f}} \sum_{i=0}^{n_{u}} \sum_{j=0}^{n_{u}} T_{i}(\xi) T_{j}(\xi)\left\{N_{u}\right\}<T_{j}^{e x t}>d \Omega \\
\left\{r_{p}\right\} & =-\int_{\Omega_{e}} \sum_{i=0}^{n_{p}} T_{i}(\xi)\left\{N_{p}\right\}\left(-(J-1)-\alpha\left(p_{h}-p_{0}\right)\right) d \Omega
\end{aligned}
$$

avec $p_{0}$ la valeur de la pression à l'instant initial $\left(t_{0}=0\right)$ telle que $\Pi\left(t_{0}\right)=0$ (si $\left.\mathbf{F}\left(t_{0}\right)=\mathbf{I}\right)$. Les tenseurs d'ordre $4 ; \mathbb{C}_{h y p}$ et $\mathbb{C}_{\text {visc }}$ étant définis à l'instant $t$ par $^{1}$ :

$$
\begin{aligned}
\mathbb{C}_{h y p}^{(t)} & =\frac{\partial \mathbf{\Pi}_{h y p}^{(t)}}{\partial \mathbf{F}(t)} \\
\mathbb{C}_{v i s c}^{(t)} & =\frac{2 \eta_{0}}{\Delta t}\left\|\dot{\mathbf{C}}^{(t)}\right\|^{n-1}\left(\frac{\partial \mathbf{F}^{(t)} \mathbf{C}^{(t)}}{\partial \mathbf{F}^{(t)}}-\mathbf{I} \oplus \mathbf{C}^{(t-1)}\right) \\
& +\frac{4 \eta_{0}(n-1)}{\Delta t}\left\|\dot{\mathbf{C}}^{(t)}\right\|^{n-3}\left(\mathbf{F}^{(t)} \dot{\mathbf{C}}^{(t)}\right) \otimes\left(\mathbf{F}^{(t)} \dot{\mathbf{C}}^{(t)}\right)
\end{aligned}
$$

Partant de cette formulation, une bibliothèque d'éléments finis réduits a été implémentée dans le code ZéBuLoN. Nous utiliserons en particulier l'élément Q9P3 (voir tableau 1).

Les éléments tridimmensionnels qui servent à éprouver la validation des éléments finis réduits sont basés sur le même formalisme et sont implémentés sur le même logiciel.

1. l'opération $A=B \oplus C$ est définie à partir de la notation indicielle suivante : $A_{i j k l}=$ $B_{i k} C_{j l}$. 


\begin{tabular}{lll}
\hline Type d'élément & T6P1 & Q9P3 \\
\hline Dimension & 3D2D & 3D2D \\
Type Géométrique & Triangle & Quadrangle \\
Interpolation des déplacements & Quadratique & Quadratique \\
Interpolation de la pression & $<1>$ & $<1, \xi, \eta>$ \\
\hline
\end{tabular}

Tableau 1. Eléments à interpolation discontinue de la pression

\section{Validation}

Afin de caractériser la qualité des solutions obtenues avec l'élément réduit, nous avons effectué des comparaisons avec un modèle de référence utilisant des éléments finis 3D classiques. Le modèle de référence est basé sur la modélisation du comportement d'un barreau élastomérique mesurant $2 \mathrm{~mm}$ de côté pour $5 \mathrm{~mm}$ de long. Ce dernier est réalisé via des éléments finis hyperélastiques de type H20P4 (éléments hexaédriques quadratiques à pression linéaire). Les paramètres matériaux du modèle viscohyperélastique ont été identifiés à partir d'essais de traction et de glissement simple, on a : $C_{10}=0.253 \mathrm{Mpa}, C_{01}=-0.06 \mathrm{Mpa}, C_{11}=0.002 \mathrm{Mpa}, C_{02}=0.0001 \mathrm{Mpa}$, $C_{20}=0.012 \mathrm{Mpa}, \eta_{0}=0.026 \mathrm{Mpa} . \mathrm{s}, n=0.28$, le coefficient de perturbation est pris de $\alpha=0.0002 \mathrm{Mpa}^{-1}$.

\subsection{Cas hyperélastique}

Nous avons réalisé sur ce modèle des simulations d'essais de traction, cisaillement ou de flexion en imposant des déplacements sur une section extrême et en encastrant l'autre extrémité. Par ailleurs, une analyse de l'influence de la finesse du maillage sur la précision des résultats du modèle de référence, a permis d'en fixer la taille, soit 39231 ddls.

Pour la validation de la réponse globale, la confrontation s'effectue à partir des composantes du torseur résultant des réactions, associées aux déplacements imposés. On utilise deux modèles réduits 3D-2D à 49 éléments de type Q9P3 pour différents ordres de la décomposition. La figure 4 présente les résultats du test de traction.

Les figures 5, 6 et 7 correspondent aux résultats des tests de cisaillement et de flexion où l'on impose un déplacement transverse ou un angle de rotation sur la partie supérieure, tout en bloquant les autres degrés de liberté. Les couplages sont très bien retranscrits par le modèle réduit, et ce avec un ordre relativement faible de la base de projection (pour $n_{u}=8$ et $n_{p}=6$ on obtient tous les couplages).

A partir d'une simple opération de post-traitement, on peut reconstruire les champs de contraintes ou de déformations d'un modèle réduit. Les figures 8 présentent une illustration qualitative de la réponse de différents modèles réduits. On peut remarquer que l'obtention d'une solution locale de bonne qualité nécessite un ordre plus important que la détermination du comportement global. 


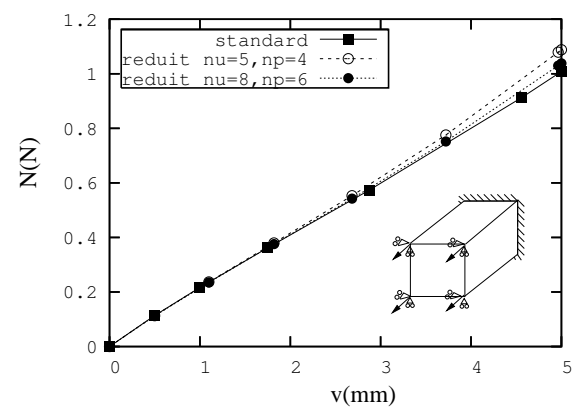

Figure 4. Test de traction (poutre d'élastomère)

Le tableau 2, récapitule les temps de calcul et les tailles de chaque modèle. Si le gain observé peut sembler assez faible pour un ordre élevé, il est facile de concevoir que dans le cas d'un barreau plus élancé le temps de calcul du modèle standard sera beaucoup plus important que celui du modèle réduit.

\begin{tabular}{lcccccc}
\hline modèle & $n_{u}$ & $n_{p}$ & d.d.l. & temps CPU (s) & gain en taille & gain en temps \\
\hline complet & & & 39231 & 1717.7 & & \\
réduit 3D2D & 5 & 4 & 3375 & 43 & 11 & 40 \\
réduit 3D2D & 9 & 8 & 6075 & 254.5 & 6 & 7 \\
réduit 3D2D & 14 & 12 & 9450 & 1014.5 & 4 & 1.7 \\
\hline
\end{tabular}

Tableau 2. Temps de calcul et taille de modèle pour un test de traction sur la poutre en élastomère

\subsection{Cas visco-hyperélastique}

Pour valider le modèle visco-hyperélastique nous avons réalisé un essai de traction cyclique en déplacement imposé. Les deux extrémités du barreau sont encastrées, le signal imposé est de type sinusoïdale avec une fréquence de $3.5 \mathrm{~Hz}$ et une amplitude de $60 \%$ de déformation.

Comme pour le cas hyperélastique, on observe un bon accord entre les résultats du modèle réduit par rapport à ceux du modèle complet, même pour un ordre faible de l'approximation $n_{u}=5, n_{p}=4$ (voir figure 9).

En termes de gain au niveau temps de calcul, le tableau 3 nous montre que même si l'on est en deçà du cas hyperélastique, le modèle réduit reste performant. 


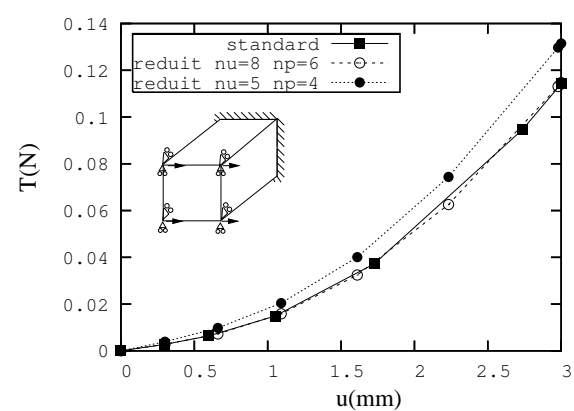

5.a résultante tangente

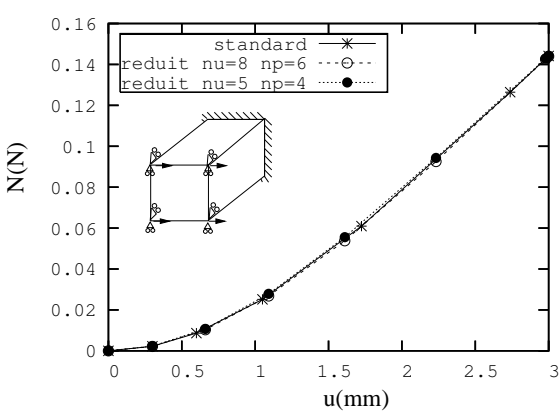

5.b résultante normale

Figure 5. Test de cisaillement, poutre d'élastomère (3D)

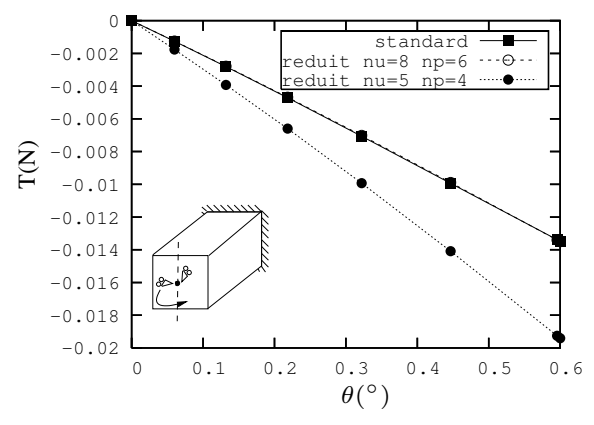

6.a résultante tangente

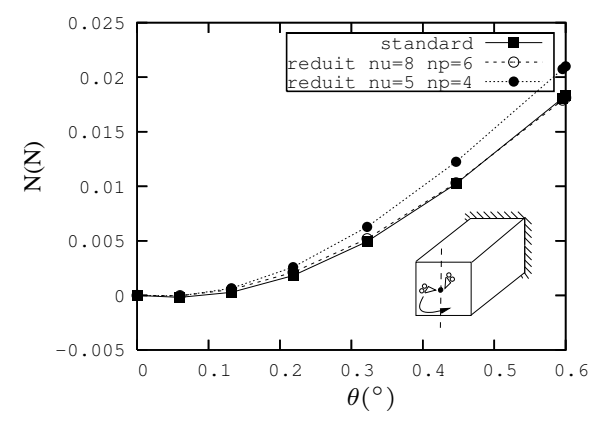

6.b résultante normale

Figure 6. Test de flexion, poutre d'élastomère (3D)

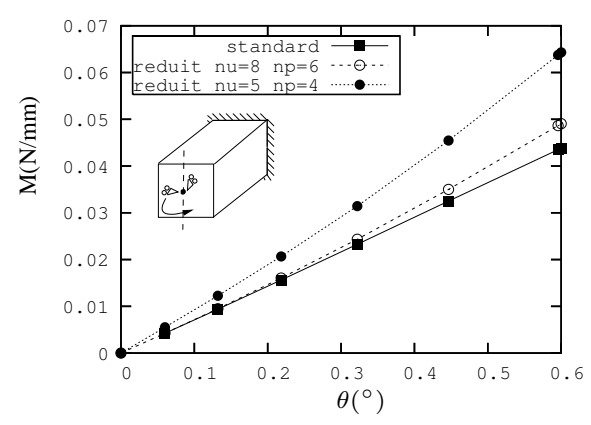

7.a Test de flexion

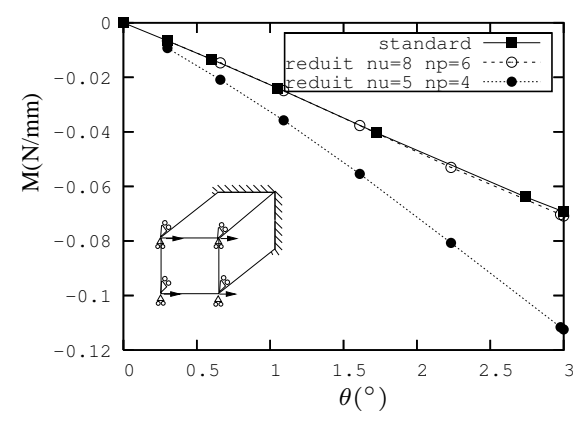

7.b Test de cisaillement

Figure 7. Moment résultant, poutre d'élastomère (3D) 

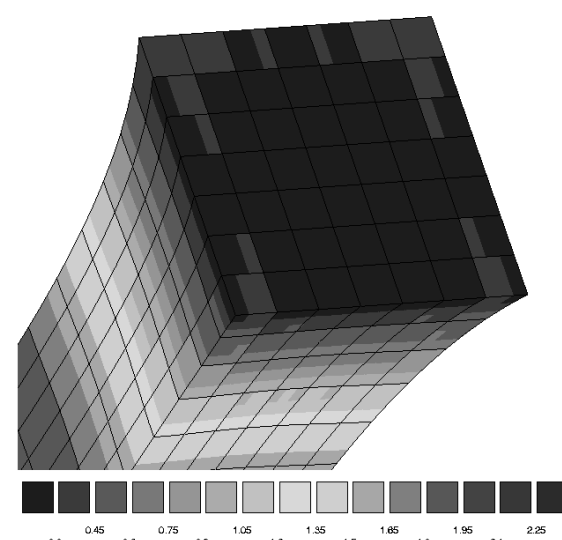

8.a Modèle réduit $n_{u}=5, n_{p}=4$

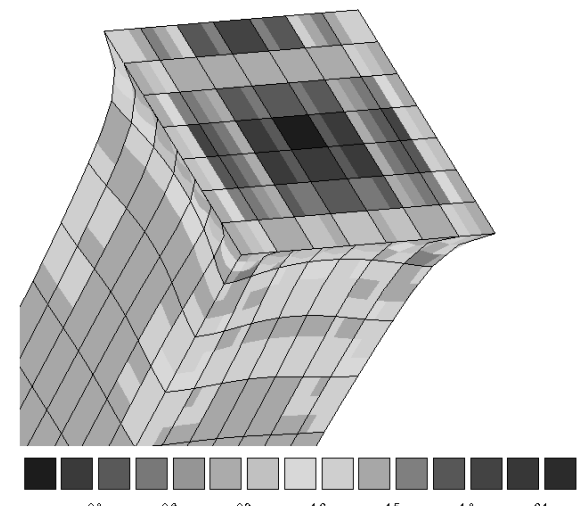

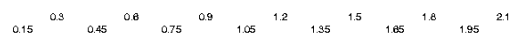

8.c Modèle réduit $n_{u}=14, n_{p}=12$
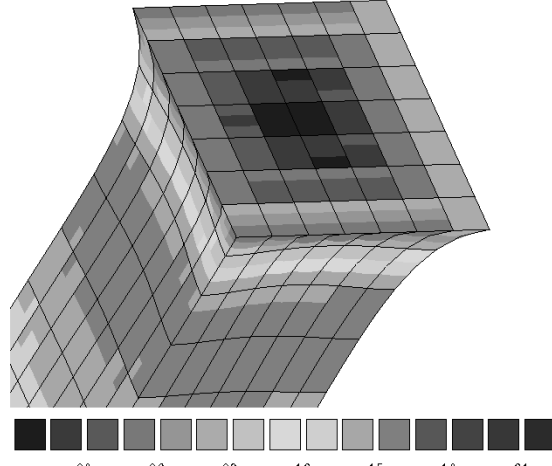

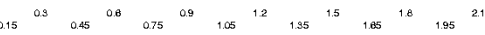

8.b Modèle réduit $n_{u}=9, n_{p}=8$

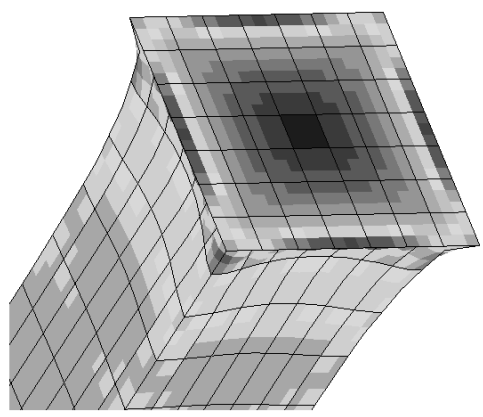

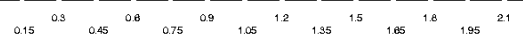

8.d Modèle de référence

Figure 8. Contrainte de Von-Mises, traction d'un bareau élastomérique

\begin{tabular}{lcccccc}
\hline modèle & $n_{u}$ & $n_{p}$ & d.d.l. & temps CPU (s) & gain en taille & gain en temps \\
\hline complet & & & 39231 & 34503 & & \\
réduit 3D2D & 5 & 4 & 3375 & 1511 & 11 & 23 \\
réduit 3D2D & 8 & 6 & 5400 & 8704 & 7.6 & 4 \\
\hline
\end{tabular}

Tableau 3. Temps de calcul et taille de modèle pour le test de traction cyclique sur la poutre en élastomère 


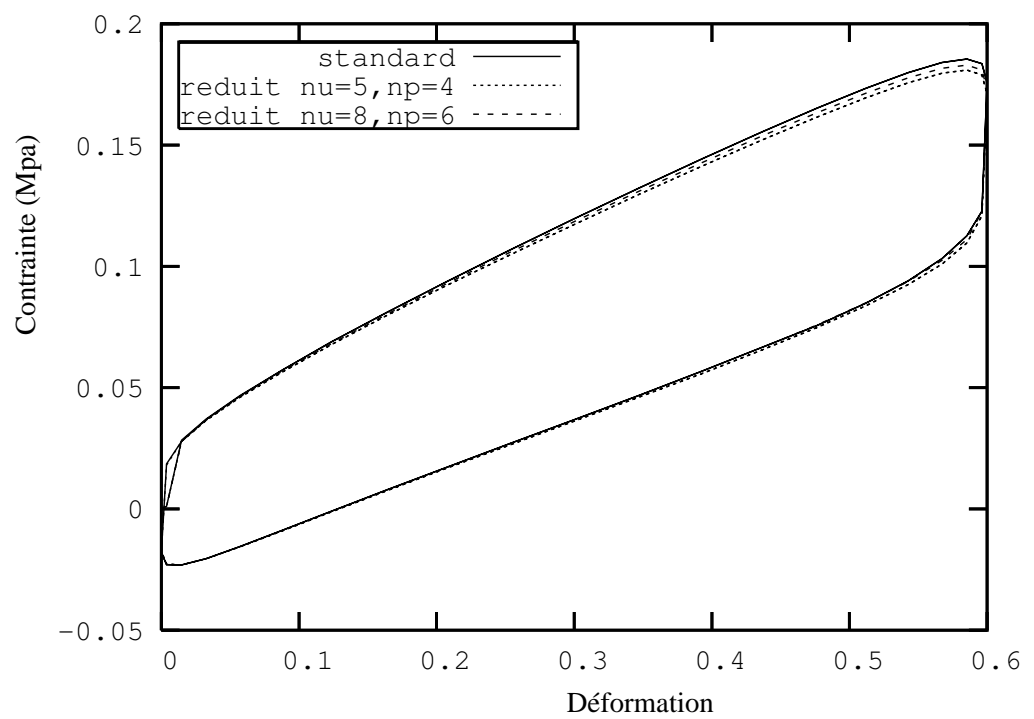

Figure 9. Traction cyclique d'un bareau élastomérique

\section{Application à la poutre E.F.B.}

La poutre composite est constituée de deux types de baguettes élastiques isotropes noyées dans une matrice élastomérique (voir figure 1). Ce type de pièce a vocation à être utilisée dans les rotors d'hélicoptères pour effectuer la liaison mécanique entre une pâle et le moyeu rotor. Ce concept novateur, doit remplacer tout un système d'articulation mécanique afin de réaliser un gain en performance, encombrement et au final en coût. La structure retenue dans cet exemple est semblable à la pièce étudiée dans les travaux de Delorme (1997) et Meo et al. (2002) (où le comportement de l'élastomère était considéré comme étant linéaire).

La section de cette structure est présentée sur la figure 10, elle a pour dimensions caractéristiques : $90 \mathrm{~mm}$ de large, $30 \mathrm{~mm}$ d'épaisseur et $750 \mathrm{~mm}$ de long. Les matériaux constituant les baguettes sont modélisés par une loi élastique linéaire isotrope, les modules ingénieurs sont présentés dans le tableau 4.

\begin{tabular}{lcc}
\hline & $E(\mathrm{Mpa})$ & $\nu$ \\
\hline matériau 1 & 55000 & 0.20 \\
matériau 2 & 125000 & 0.25
\end{tabular}

Tableau 4. Caractéristiques ingénieurs des matériaux 1 et 2 


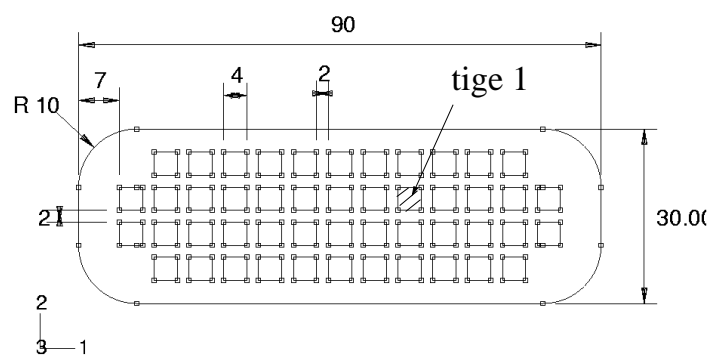

Figure 10. Section de la poutre composite E.F.B.

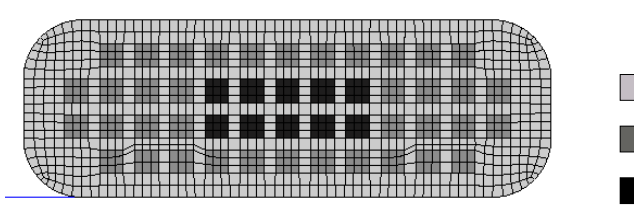

élastomère

matériau 1

matériau 2

Figure 11. Maillage de la section en éléments réduits 3D-2D (type Q9P3)

Le cas de charge traité correspond à un essai de traction-cisaillement combiné. Une section extrême de la poutre est encastrée, l'autre est soumisse à un déplacement de $4 \mathrm{~mm}$ dans la direction de traction puis à un déplacement cyclique de $5 \mathrm{~mm}$ dans la direction transverse (suivant $X$ figure 1). Le signal cyclique imposé est de type triangulaire avec une fréquence de $3.5 \mathrm{~Hz}$

La section de la structure est maillée avec 1117 éléments réduits de type Q9P3 (cf. figure 11). Avec $n_{u}=5, n_{p}=4$ le modèle réduit comprend 69255 degrés de liberté. A titre de comparaison, si l'on devait considérer un maillage tridimensionnel de la structure, ayant la même taille élémentaire que le maillage réduit proposé et un élancement élémentaire acceptable (de l'ordre de 5), on obtiendrait plus de 5 millions de degrés de liberté.

La figure 12.a présente la réponse globale de la structure pour le cas de chargement décrit précédemment. Après post-traitement du modèle réduit, on peut visualiser la déformée globale de la structure, obtenue en cisaillement (cf. figure 12.b avec un facteur amplificateur de 10). Les figures 13.a et 13.b montre la contrainte de VonMises dans les tiges renforçantes. De la même manière que pour le cas hyperélastique (figures 8), il semble que l'ordre de la base soit insuffisant pour tenir finement compte des effets de bords, on peut néanmoins obtenir une estimation locale des contraintes avec un temps de calcul raisonnable. L'introduction de fonctions de forme spécifiques (non polynomiales) pourrait permettre de palier ce problème. 

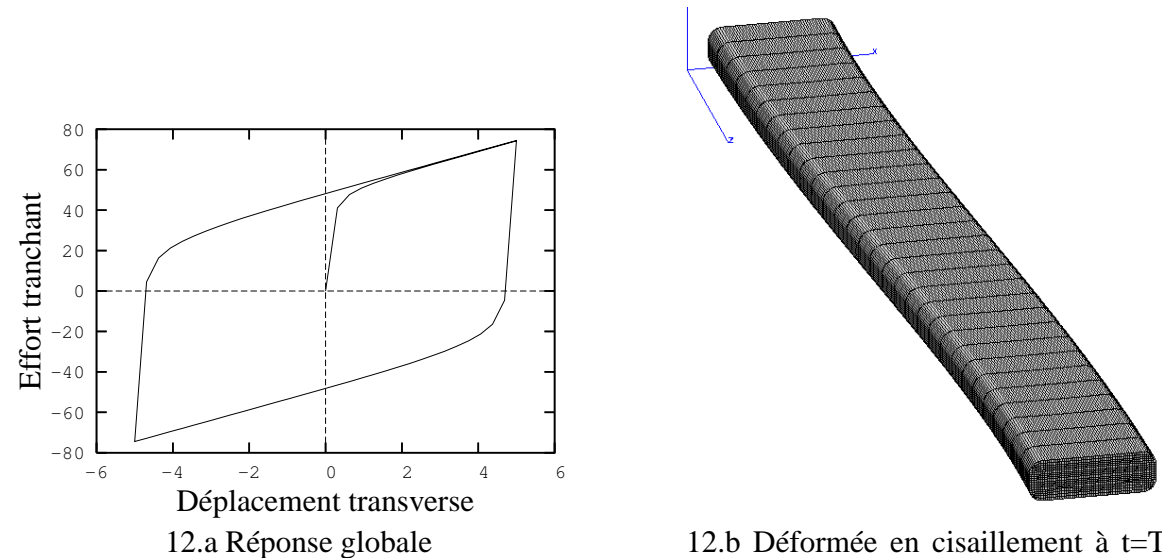

12.b Déformée en cisaillement à $\mathrm{t}=\mathrm{T} / 4$ (avec un facteur amplificateur de 10)

Figure 12. Cisaillement cyclique de la poutre E.F.B. (avec un préchargement de traction)

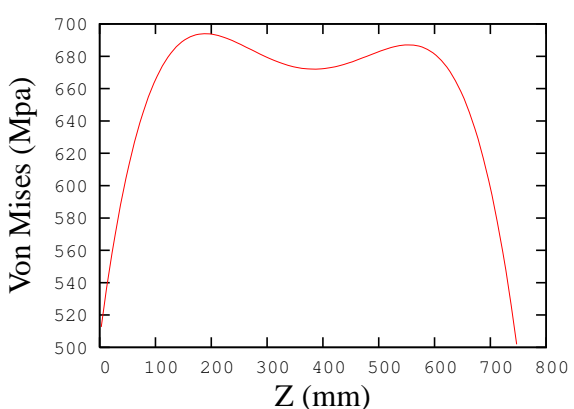

13.a évolution le long de la baguette 1 (voir figure 10)
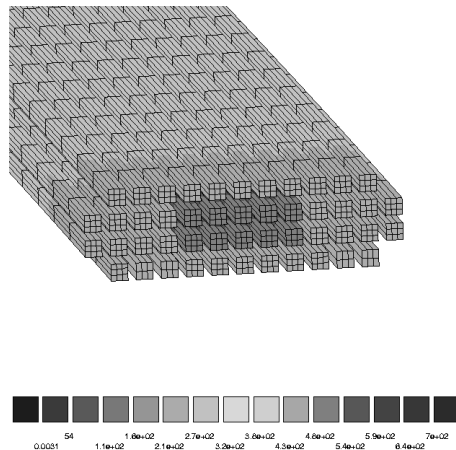

13.b isocouleur aux extrémités des tiges renforçantes

Figure 13. Visualisation de la contrainte de Von-Mises dans les tiges renforçantes (à $t=T / 4)$

\section{Conclusion}

Nous avons proposé dans ce papier, une technique de réduction de modèles à comportement non linéaire, permettant de traiter le cas de structures possédant une propriété d'invariance suivant une direction et dont l'analyse directe conduit généralement à un grand nombre de degrés de libertés ou un rapport d'élancement d'éléments pénalisants lors d'une modélisation éléments finis classique. Ainsi, à l'aide d'une projection des champs inconnues sur une base polynomiale d'ordre suffisamment élevé, on peut condenser une direction géométrique et ainsi réduire la dimension du modèle. 
Une bibliothèque d'éléments réduits a ainsi été réalisée, pour résoudre des problèmes de structures à matrice élastomérique quasi incompressible. Les cas hyperélastiques et visco-hyperélastiques (de type Kelvin-Voigt à viscosité non linéaire) ont ainsi été validés.

Cette technique permet de déterminer, avec un gain important sur le nombre de degrés de liberté, la réponse globale de structures élancées, tout en déterminant de manière assez précise les états de contraintes et de déformations locales. Cependant, il semble que cette méthode pourrait être améliorée afin de mieux prendre en compte les conditions aux bords, via un choix particulier des fonctions de formes (non polynomiales).

\section{Bibliographie}

Boukamel A., Etude théorique et expérimentale d'un stratifié caoutchouc-acier en grandes déformations, Thèse de doctorat, Université d'Aix-Marseille II, 1988.

Cheung Y., « The finite strip method in the analysis of elastic plates with two opposite simply supported ends », Institution of Civil Engineers Proceedings, vol. 40, p. 1-7, 1968.

Cheung Y., Au F., « Isoparametric Spline Finite Strip for Degenerate Shells », Thin-Walled Structures, vol. 21, p. 65-92, 1995.

Cheung Y., Jiang C., « Finite layer method in analyses of piezoelectric composite laminates », Computer methods in applied mechanics and engineering, vol. 191, p. 879-901, 2001.

Choi C., Kim K., Hong H., « Spline finite strip analysis of prestressed concrete box-girder bridges », Engineering Structures, vol. 24, p. 1575-1586, 2002.

Cugnon F., Automatisation des calculs éléments finis dans le cadre de la méthode-p, Thèse de doctorat, Université de Liège, 2000.

Davenne L., Ragueneau F., Mazars J., Ibrahimbegocvic A., « Efficient approaches to finite element analysis in earthquake engineering », Computers \& Structures, vol. 81, p. 12231239, 2003.

Delorme D., Modélisation numérique du comportement mécanique de matériaux composites à matrice élastomèrique, Thèse de doctorat, Université d'Aix-Marseille II, 1997.

Devries F., « Homogenization of elastomer matrix composites : method and validation », Composites Part B : Engineering, vol. 29, p. 753-762, 1998.

Dumontet H., Homogénéisation et effets de bords dans les matériaux composites, Thèse d'état, Université Pierre et Marie Curie Paris 6, 1990.

Duster A., Hartmann S., Rank E., « p-FEM applied to finite isotropic hyperelastic bodies », Comput. Methods Appl. Mech. Engrg., vol. 192, p. 5147-5166, 2003.

Herrmann L. R., « Elasticity equations for incompressible and nearly incompressible material by a variational theorem », AAIA J., vol. 3, p. 1896-1900, 1964.

James A., Green A., Simpson G., « Strain energy functions of rubber. I. Characterization of gum vulcanizates. », Journal of Applied Polymer Science, vol. 19, p. 2033-2058, 1975.

Lahellec N., Mazerolle F., Michel J., « Second-order estimate of the macroscopic behavior of periodic hyperelastic composites : theory and experimental validation », Journal of the Mechanics and Physics of Solids, vol. 52, p. 27-49, 2003. 
Lejeunes S., Boukamel A., Cochelin B., « Model reduction method : an application to the buckling analysis of laminated rubber bearings », Revue Européenne de Mécanique Numérique, vol. 15/1-2-3, p. 281-292, 2006.

Léné F., Rey C., « Some strategies to compute elastomeric lamified composite structures », Composite Structures, vol. 54, p. 231-241, 2001.

Meo S., Debordes O., Boukamel A., « Assemblage de structures une à une invariantes dans une direction », Mécanique \& Industries, vol. 3, p. 211-225, 2002.

Rivlin R. S., Saunders D. W., « Large elastic deformations of isotropic materials VII. Experiments on the deformation of rubber », Philosophical Transactions of the Royal Society of London., vol. 243, p. 251-288, 1951. 
